\title{
THE APPLICATION OF ZIGBEE PHASE SHIFT MEASUREMENT IN RANGING
}

\author{
Jacek RAPIŃSKI
}

Institute of Geodesy, University of Warmia and Mazury in Olsztyn, Poland

*Corresponding author's e-mail: jacek.rapinski@uwm.edu.pl

\begin{tabular}{l} 
ARTICLE INFO \\
\hline Article history: \\
Received 18 February 2015 \\
Accepted 13 April 2015 \\
Available online 29 April 2015 \\
\hline Keywords: \\
Ranging \\
Indoor positioning \\
ZigBee
\end{tabular}

\begin{abstract}
This paper describes the initial assessment of the phase shift measurement unit embedded in a ZigBee communication device. Such a device can provide a very good base for a range-based indoor positioning or proximity detection system. The paper gives a description of the device and shows the results of test measurements. Tests were performed in various conditions: multipath free, outdoor environment, severe multipath indoor environment and dynamic environment with moving transceiver. The examples of results are depicted and conclusions are drawn. The phase shift measurement technique in the radio frequency (RF) network can increase the use of this networks in the area of localization based services.
\end{abstract}

\section{INTRODUCTION}

The need for localization based services has grown rapidly in a recent few years. While in the outdoors environment it is almost fulfilled with the GNSS systems, the localization indoors is still being developed. Indoor navigation can be based on various physical phenomena. The research is being performed on the use of MEMS inertial navigation, computer vision, sonar and radar systems, laser systems and various radio frequency (RF) systems. In the area of RF systems the group of methods connected to communication networks are of special importance. Their main advantage is that they can use existing communication signals (such as Bluetooth, Wi-Fi or ZigBee) for positioning. In this paper the main focus is on ZigBee protocol and a newly introduced ranging method.

Positioning in wireless networks can be made using two approaches. First one is so called range-free approach. It is based on the received signal strength indicator (RSSI) (Chen et al., 2012).

In this approach the location of user is estimated on the basis of the map of previously measured signal strength. This method is easy to implement and available for all protocols, but it has low accuracy. The second approach is range-based approach, in which measured distances and multilateration are used for position estimation. The distances are usually measured using time of flight principle or they are estimated on the basis of RSSI. The new approach to ranging in ZigBee network is phase shift measurement.

ZigBee is a specification of a transmission protocol in wireless mesh or cluster tree networks for low-cost IEEE 802.15.4 devices. IEEE 802.15.4 defines a standard for wireless communication on the level of physical layer (PHY) and medium access control (MAC) layer. ZigBee specification adds a network layer (NWK) and application layer (APL) on top of IEEE 802.15.4 (Figure 1).

While IEEE 802.15.4 is developed and maintained by the Institute of Electrical and Electronics Engineers, the ZigBee is maintained by the ZigBee Alliance (zigbee.org). ZigBee networks are very energy efficient - single device can operate for months or even years on a single alkaline battery. The main disadvantage of ZigBee networks is low transmission speed (up to $250 \mathrm{kbps}$ ). On the other hand these networks can work in any type of network topology with maximum number of 65,000 nodes. The range of operation is between 10 and $300 \mathrm{~m}$.

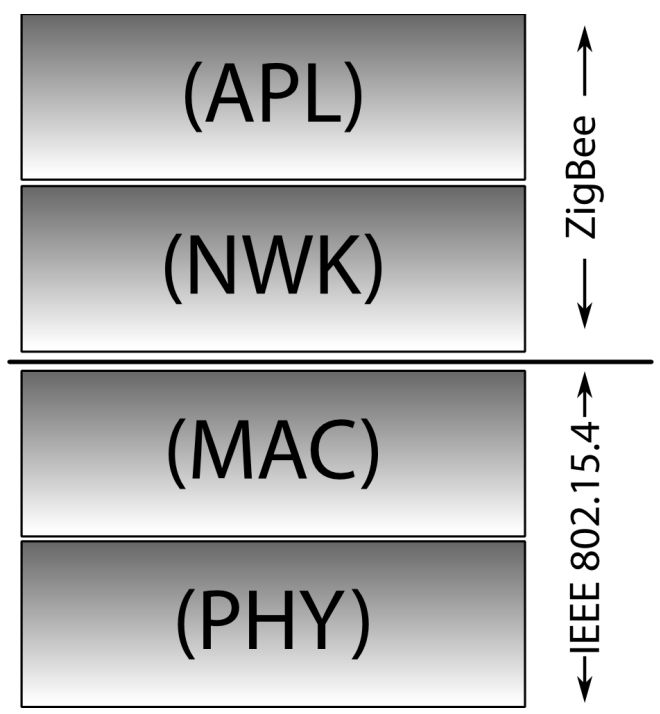

Fig. 1 Full ZigBee stack. 


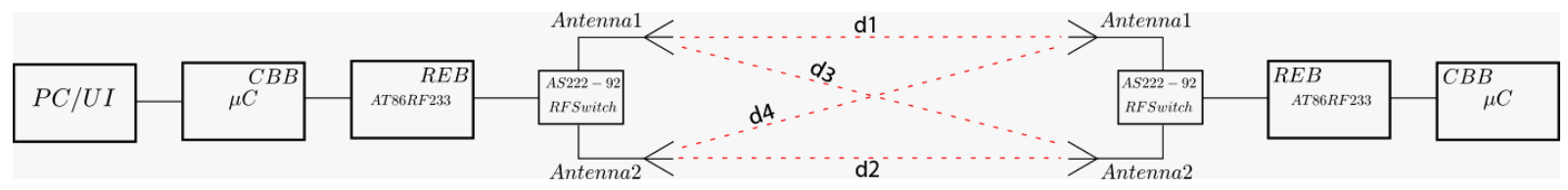

Fig. 2 Outline of REB233SMAD-EK.

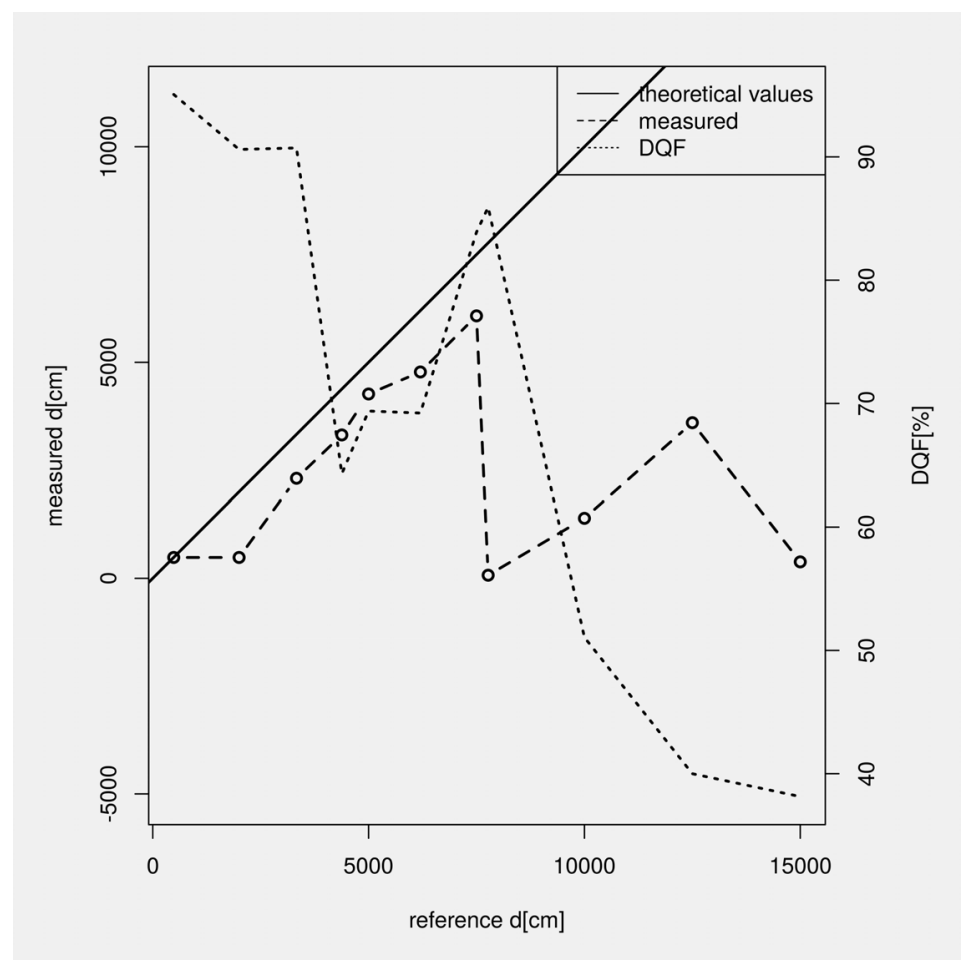

Fig. 3 Distance in [cm] as a result of mean PMU readings and DQF vs. Distance.

Typical application of this protocol are sensor networks, personal networks (WPAN), home automatic, alarm systems or monitoring systems. ZigBee specification for MAC and PHY layers ensures transmission in $868 \mathrm{MHz}, 915 \mathrm{MHz}$ and $2.4 \mathrm{GHz}$ band. It is using BPSK modulation of $868 / 915 \mathrm{MHz}$ bands and O-QPSK modulation of $2.4 \mathrm{GHz}$.

The ZigBee signal can be used for indoor navigation. There are a lot of studies describing positioning using RSSI (Received Signal Strength Indication), angle of arrival (AOA) (Peng and Sichitiu, 2006) or TOF (Time of Flight) (Disha 2013). Recently, the new (for a ZigBee) distance measurement technology was introduced in AT86RF233 chip (Smieja, 2014).

\section{DEVICE DESCRIPTION}

To investigate the possibility and performance of this new (in the area of RF indoor positioning) measurement technique, the REB233SMAD-EK evaluation kit was used. The kit consists of two controller base boards (CBB) equipped with ATxmega256A3 micro-controllers and two radio extend boards (REB) with AT86RF233 chips. Each REB is equipped with two SMA mounted antennas.
The signal is switched between these antennas using AS222-92 RF switch. The outline of the device is presented in Figure 2.

In each measurement epoch four distances (namely $\mathrm{d} 1, \mathrm{~d} 2, \mathrm{~d} 3$ and $\mathrm{d} 4$ ) are measured using antenna diversity (also known as space diversity). Antenna diversity is a way of mitigation of the multipath-caused problems such as phase shifts, time delays, distortions or attenuations. In this case the spatial diversity with two physically separated antennas is used.

The quality of the received signal is expressed by the distance quality factor parameter (DQF). This parameter intends to describe the quality of received measurement, but it is not described in the data-sheet.

According to the datasheet, Phasor Measurement Unit (PMU) results are stored in the PHY_PMU_VALUE. This is an 8-bit PMU measurement register updated every $8 \mu$ s. The experiment was performed in three scenarios: stationary outdoors, stationary indoor and dynamic outdoor. In addition the behavior of PMU measurements at long range was tested. The main goal of this experiments was to estimate maximum range and quality of distance measurement in various conditions. 

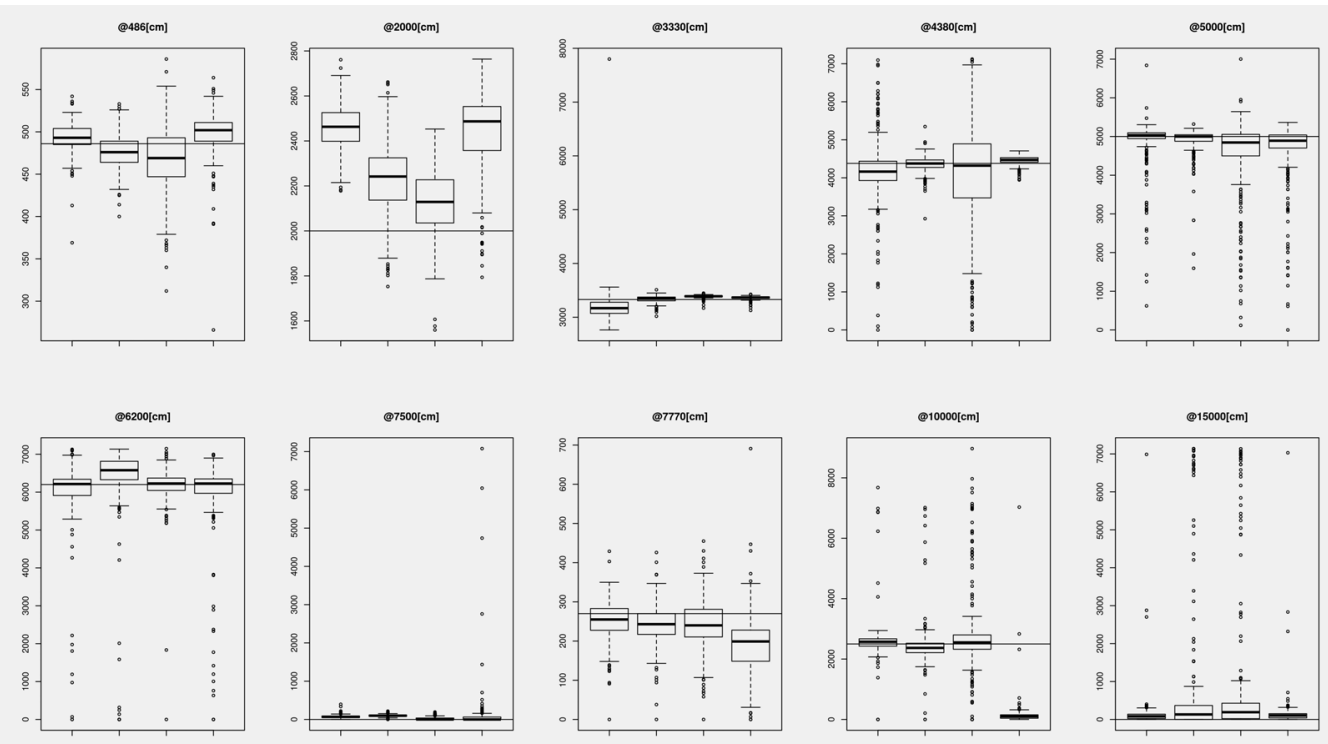

Fig. 4 Distribution of the PMU readings at various distances.

\section{RESULTS}

In this section the results depicting the performance of distance measurements are presented in three subsections.

\subsection{MEASUREMENT RESULTS VERSUS TRUE DISTANCE}

Figure 3 depicts the dependency between measured distance, reference distance and DQF. For this figure the results from all pairs of antennas were averaged (distances and DQFs). Continuous line is a reference (true distance vs. true distance). The dotted line and right side axis depicts the change in DQF. It is degrading almost linear with increasing distance with one "peak" at $75 \mathrm{~m}$. The dashed line depicts the PMU readings vs. distance. It can be noticed, that at about $75 \mathrm{~m}$ and $150 \mathrm{~m}$, the PMU reading is "reset" to 0 . It indicates that this is the maximum range that can be directly measured with this device. All distances longer then this are burdened with a certain "ambiguity". On the basis of these results, we can assume that the PMU is operating on the intermediate frequency (which is mentioned in the data-sheet but not documented).

The distribution of the PMU measurements at various distances is depicted in Figure 4 in the form of box plots. In each subfigure one box depicts one pair of antennas. Top and bottom of the box represent first and third quartiles, the band inside the box is second quartile. The whiskers depicts the lowest datum still within 1.5 interquartile range of the lower quartile, and the highest datum still within 1.5 interquartile range of the upper quartile.

Analyzing Figure 4 it can be noticed that antenna diversity can improve the overall performance of the device. For example at $100 \mathrm{~m}$ one pair of antennas gives totally wrong results. In all of the cases the results and its standard deviations varies between antennas, with at least one result close to the correct range. The subfigure presenting readings at $75 \mathrm{~m}$ shows that for one pair of antennas single reading were crossing the full wavelength hence the readings oscillates between 0 and 7500 .

Another important aspect of the signal is its noise characteristics. Since PMU results for all of the antennas should reflect the same distance, and DQF should reflect the quality of each signal (it is supposed to be used for weighting), the correlation between this values should be investigated. Correlation matrix presented in Figure 5 shows, that there is no significant correlation between any of presented values. It can mean that the noise in the data is nondeterministic.

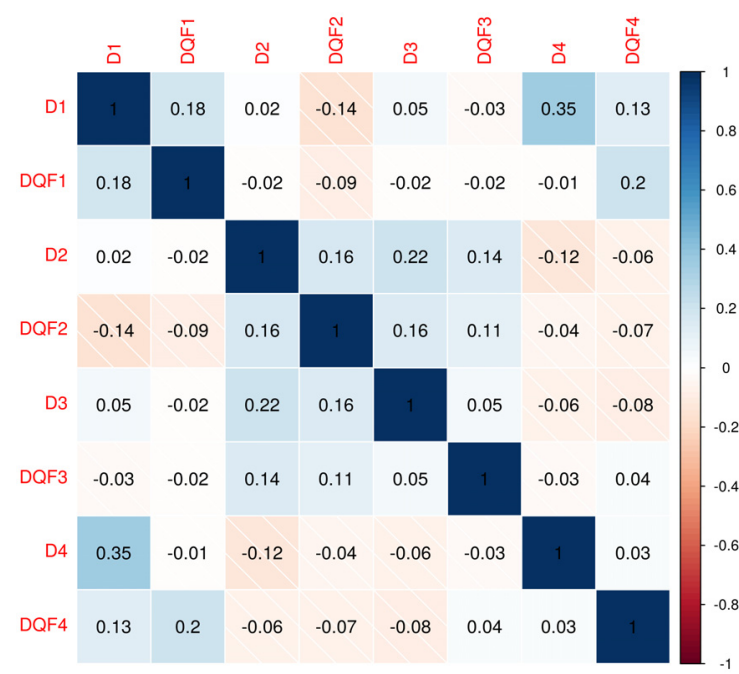

Fig. 5 Correlation matrix. 
Indoor survey @ 290 cm, severe multipath

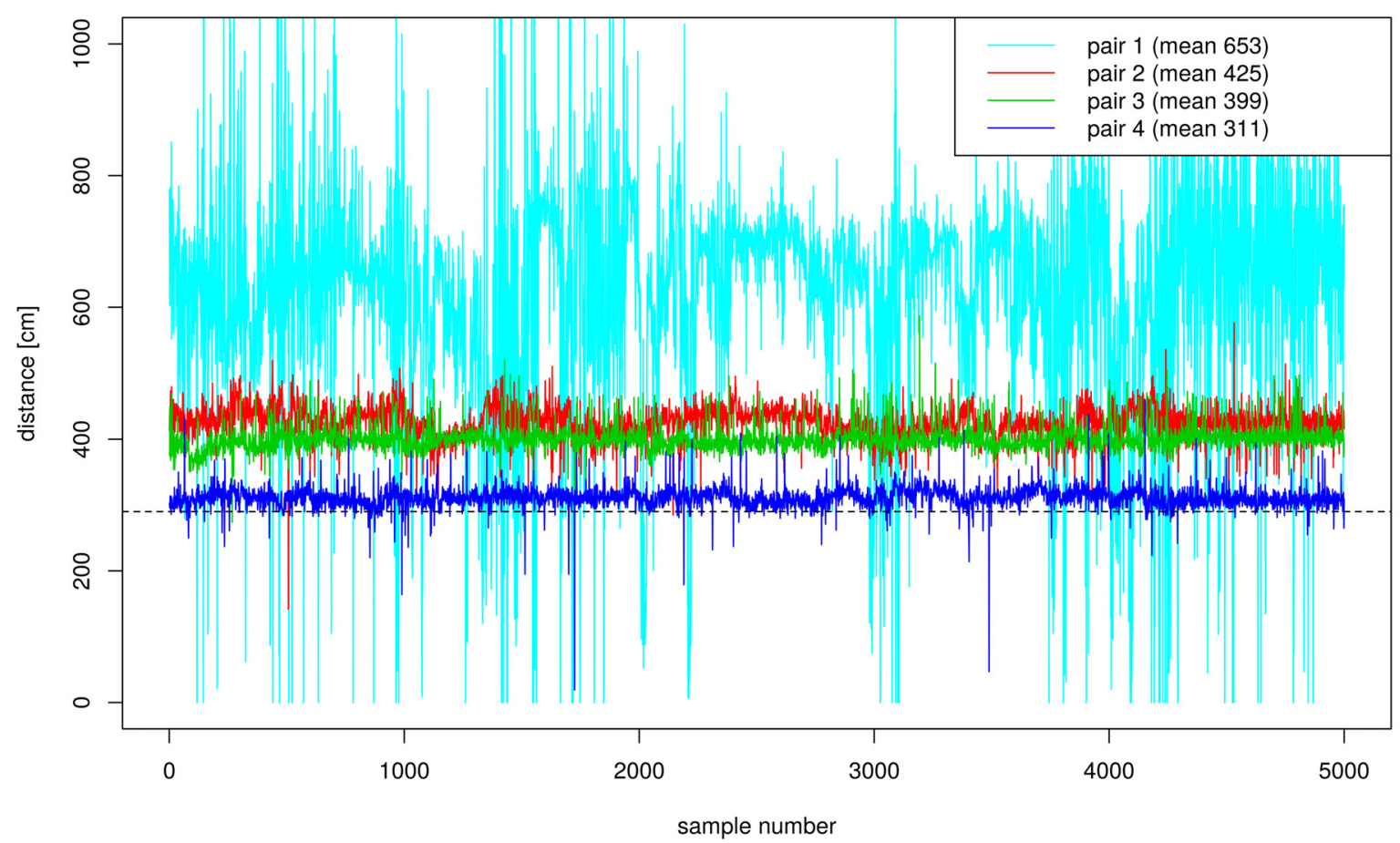

Fig. 6 Results in the indoor environment.
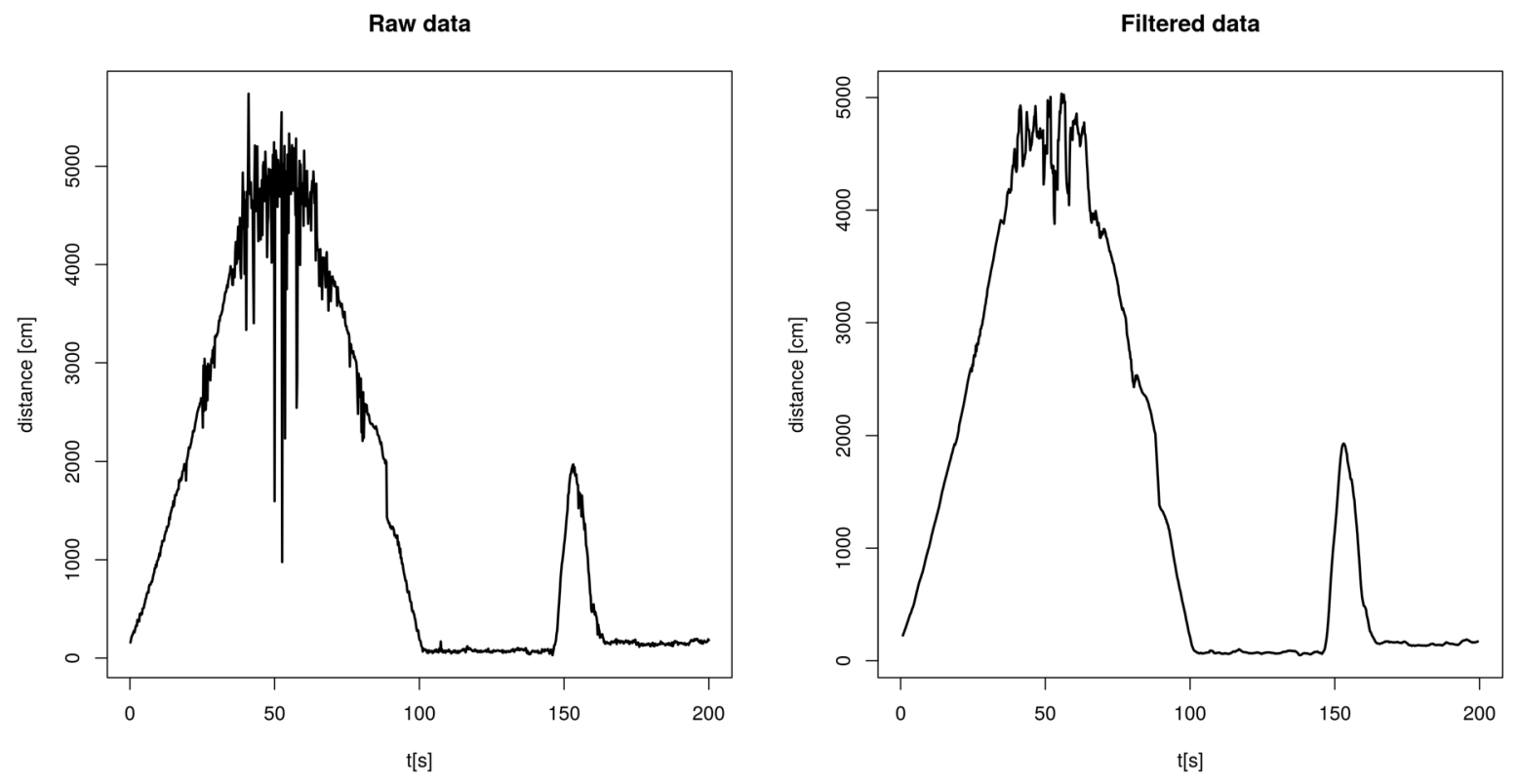

Fig. 7 Results of test with moving transceiver.

\subsection{INDOOR ENVIRONMENT}

The test in the indoor environment was conducted in a small room. Reference distance was $290 \mathrm{~cm}$ in a severe multipath environment. The results are presented in Figure 6.

The results for pair 4 are closest to the reference distance - the mean distance is $21 \mathrm{~cm}$ which is equal to $7 \%$ relative error. Pairs two and three are slightly more distant from the reference distance $(37 \%$ and

$46 \%$ relative error). Results for pair one gave $125 \%$ relative error.

\subsection{MOVING RECEIVER}

The last test was made when one of the nodes was moving. The transceiver was moved from 0 to 50 meters, then it was stopped and returned to 0 . After a while it was moved $20 \mathrm{~m}$ and returned without stopping. The results are depicted in Figure 7. The left 
subfigure depicts raw data, while right subfigure depicts the same data smoothed with a 7 tap moving average. A more sophisticated algorithm for data smoothing is presented in (Rapinski and Smieja, 2015). The results reflects the movement in a proper way. The noise level increases with distance and is at the maximum at the $50 \mathrm{~m}$ distance when the transceiver was stationary for 20 seconds.

\section{DISCUSSION}

The phase measurement unit embedded into AT86RF233 chip provides ranging capabilities using RF band. From the preliminary tests, the performance of ranging is much better than RSSI or TOF techniques commonly used in indoor positioning. In the experiments the device was operating at $2.4 \mathrm{GHz}$ band. "Resetting" of distance to 0 at certain distances suggests that the phase measurement is conducted on intermediate frequency. Since there is no significant correlation between distances and their corresponding DQF's, an insightful investigation is required to determine if DQF can be used for example for weighting of distances. While measuring in the dynamic mode (one of the nodes was moving), there noise is growing with distance but the movement dynamics is reflected properly.

In the indoor environment, the multipath effect is clearly visible. Only one pair of antennas is returning results correctly and is probably reflecting the line of sight propagation. Distribution of measurement results shows that the number of outliers is increasing with distance. At $150 \mathrm{~m}$ and $75 \mathrm{~m}$ extremely large outliers are caused by oscillation around distances "reset" point.

The ranging performance introduced by this chip encourages for further research and use of this device in positioning and indoor navigation applications. An important benefit of such a solution is combination of positioning and communication functionality in one, very energy efficient device.

\section{REFERENCES}

Adalja Disha, M.: 2013, A comparative analysis on indoor positioning techniques and systems. International Journal of Engineering Research and Applications, 3(2), 1790-1796.

Chen, Q., Liu, H., Yu, M. and Guo, H.: 2012, RSSI ranging model and $3 \mathrm{~d}$ indoor positioning with ZigBeenetwork. In Position Location and Navigation Symposium (PLANS), IEEE/ION, 1233-1239.

DOI:10.1109/PLANS.2012.6236979
Peng, R. and Sichitiu, M.: 2006, Angle of arrival localization for wireless sensor networks. In 2006 3rd Annual IEEE Communications Society on Sensor and Ad Hoc Communications and Networks, SECON '06, 1, 374-382. DOI:10.1109/SAHCN.2006.288442

Rapinski, J. and Smieja, M.: 2015, ZigBee ranging using phase shift measurements. Journal of Navigation, available on CJO2015. DOI: $10.1017 / \mathrm{S} 0373463315000028$

Smieja, M.: 2014, The use of RF communication devices in ranging applications. The 9th International Conference "ENVIRONMENTAL ENGINEERING" 22-23 May 2014, Vilnius, Lithuania. Available online at http://enviro.vgtu.lt 